\title{
I6. COMMISSION POUR LES OBSERVATIONS PHYSIQUES DES PLANETES ET DES SATELLITES
}

\author{
Président: M. M. Maggini, Direttore del R. Osservatorio Astronomico, Collurania, \\ Teramo, Italia.
}

Membres: MM. Andrea, Antoniadi, Armellini, L. Campbell, Delporte, Dunham, Hargreaves, Mlle Harwood, MM. Jarry-Desloges, Lampland, Link, LuplauJanssen, Lyot, Nicholson, Peek, Phillips, Plakidis, Quénisset, F, E. Ross, E. C. Slipher, Wildt, F. E. Wright, W. H. Wright.

Depuis la dernière Assemblée générale, notre commission a eu le regret de perdre deux de ses membres les plus éminents: M. Paul Stroobant, directeur de l'Observatoire Royal de Belgique et M. W. H. Pickering, ancien directeur des succursales de l'Observatoire de Harvard à Arequipa (Pérou) et Mandeville (Jamaïque). Les recherches de M. Paul Stroobant se rapportent pour une grande partie aux planètes, notamment Mercure, Vénus, les petites planètes et Saturne dont il étudia pendant des années les anneaux. Il faisait partie de notre Union comme président de la $5^{\circ}$ commission et membre de beaucoup d'autres.

M. William H. Pickering, frère de E. C. Pickering, le savant directeur de l'Observatoire de la Harvard University, mort en I9I9, était, lui aussi, un des astronomes les plus connus. Observateur passionné de la planète Mars, d'abord à Arequipa et Flagstaff, ensuite à la Jamaïque, il avait organisé un groupe d'observateurs de la planète Mars, "Associated observers of Mars", dont il publiait l'analyse des travaux dans ses "Reports on Mars" qui paraissaient dans Popular Astronomy. On doit à W. H. Pickering la découverte de deux satellites de Saturne.

\section{Observations des Surfaces PLANÉtaires}

Mercure. Deux travaux dus à McEwen ont paru dans B.A.A.J. 46 et 47. Le premier est une analyse des cartes générales de la planète parues depuis 1889 , dues à Schiaparelli (I889), Lowell (I897), aux observations de M. Jarry-Desloges (I907-20), Antoniadi (I929), McEwen (1929); ces cartes ont été réduites par McEwen à la même échelle dans la projection de Mollweide.

Le deuxième travail se rapporte à l'élongation orientale de avril-mai I936 et résume les observations de quatre observateurs. La comparaison de la courbe théorique de phase avec la courbe tracée d'après les observations a révélé dans cette dernière des irrégularités qu'on ne peut pas expliquer par des fautes de mesure.

Vénus. E. M. Antoniadi a donné dans $L^{\prime}$ Astronomie une vue d'ensemble de nos connaissances sur Vénus ainsi qu'un résumé des observations qu'il a effectuées à Juvisy et à Meudon. Il en déduit une rotation très lente qui paraît se chiffrer plutôt par mois que par semaines, sans que cela exclue la possibilité de l'identité des périodes de rotation et de révolution autour du Soleil.

A l'Observatoire Astronomique de l'Université d'Athènes, sous la direction de M. Plakidis, la planète Vénus a été observée en 1935 et r937 par J. E. Focas. On a observé plusieurs taches blanches irrégulières, séparées par des lignes et des taches sombres assez stables.

Une étude sur le terminateur de Vénus, fondée sur 900 mesures, a été publiée par McEwen dans B.A.A.J. 48.

Mars. R. L. Waterfield a publié (B.A.A.J. 45) le rapport sur l'opposition I935, observations effectuées par les membres de la British Astronomical Association. Ce rapport contient trois cartes générales de la planète, dressées d'après les 
observations des membres de la B.A.A. ou bien d'après M. Antoniadi et M. Barker. M. Waterfield a ajouté à son rapport quelques considérations sur la nature des "canaux" qu'il considère comme une manière de représentation d'un ensemble de taches. Il rappelle qu' un progrès réel dans l'Aréographie ne s'obtiendra quelorsqu'on étudiera surtout les taches principales, laissant de côté les aspects fugitifs et faux présentés par les menus détails.

Une opposition de la planète a eu lieu le I9 mai 1937. Des observations ont été effectuées à l'Observatoire de l'Université d'Athènes par J. E. Focas. On a noté les changements de couleur des taches ainsi que l'intensité des traînées sombres. Un assez grand nombre de nuages blancs brillants a été observé, surtout dans l'hémisphère austral qui était en plein hiver; on a remarqué aussi des voiles diffus qui masquaient les régions sombres.

Cette même opposition a été observée par plusieurs membres de la Société Astronomique de France ( $L^{\prime} A$ stronomie, I937). E. C. Slipher a publié une note sur un phénomène d'origine atmosphérique observé sur Mars (Publ. Astr. Soc. Pac. 49).

Jupiter. T.'E. R. Phillips a publié dans les Mémoires de la B.A.A. le $28^{e}$ rapport de la section de Jupiter contenant la discussion des observations effectuées par les membres de la section pendant les oppositions I929-30 et I930-3I. Parmi les caractéristiques présentées par l'opposition r929-30 il faut rappeler l'apparition sur la zone tempérée nord de petites taches animées par une vitesse de rotation rapide avec une période de $9^{\mathrm{h}} 54^{\mathrm{m}}$. Il faut rappeler aussi le mouvement anormal montré par les taches de la composante australe de la bande tempérée nord, mouvement montré aussi pendant les oppositions I880 et I89I. Cette opposition I929-30 a été remarquable à cause du caractère du déplacement des taches dans le courant tropical nord.

L'opposition r930-3I a été analysée, elle aussi, par M. Phillips, surtout pour ce qui se rapporte à la région de la tache rouge et à la période de rotation qui correspond aux bandes sombres.

L'étude des changements de couleur de la zone équatoriale a été continuée par Stanley Williams, McIntosh et d'autres. M. Phillips a analysé les résultats obtenus par McIntosh; il pense que l'état de turbulence dans la bande équatoriale sud joue un rôle important dans les changements de couleur de la zone équatoriale (B.A.A.J. 46).

Pour ce qui se rapporte aux trois dernières oppositions, T. E. R. Phillips a continué l'observation systématique des passages des taches au méridien central du disque pour la détermination de la période de rotation.

B. M. Peek, le directeur actuel de la Section de Jupiter de la B.A.A. nous écrit que son travail a été la réduction des observations de longitude dans le but d'en déduire les périodes de rotation des taches. Les données se rapportant à l'opposition I933 sont complétées; on a commencé la réduction des mesures de I934.

M. Peek a publié aussi un article dans The Observatory, 59 (I936) dans le but de donner les renseignements aux observateurs qui veulent étudier la surface de Jupiter.

Saturne. Dans son Rapport de la Section de Saturne de la B.A.A., M. Phillips donne un résumé des observations effectuées par les membres lors de la disparition de l'anneau en r936-37. L'anneau de crêpe a été vu par M. Phillips plusieurs fois, ainsi que la division de Cassini. La partie de l'anneau qui était projetée sur le globe de la planète a paru double jusqu'à mi-septembre I936; elle est devenue plus difficile à voir parce que la luminosité de l'anneau était égale à celle des régions équatoriales. 
A l'Observatoire de Strasbourg MM. Danjon et Rougier on pu suivre la planète surtout pendant la période du 29 décembre I936 au 21 février I937, lorsque les anneaux nous montraient leur face opposée au Soleil. L'anse précédente ou occidentale est restée pendant tous ces mois plus brillante que l'autre; elle était visible jusqu'au bord de la planète, tandis que l'anse suivante paraissait nettement détachée du globe. L'anneau a été visible pendant toute la période d'observation (L'Astronomie, 5I, I927).

E. M. Antoniadi a, lui aussi, constaté la visibilité de l'anneau pendant le mois de janvier I937; il n'a jamais vu Saturne dépourvu de son anneau pendant les années I936-37. Pour ce qui se rapporte au globe, M. Antoniadi fait remarquer la différence qu'il y a entre les taches sombres qu'on voit sur Saturne et celles qu'on voit sur Jupiter: tandis que les taches de Jupiter sont disposées le long des marges des bandes, celles de Saturne sont placées dans les bandes (B.A.A.J. 47).

La visibilité de l'anneau pour ces positions dans lesquelles il aurait dû être sombre, a été analysée par Ph. Fauth et J. Dieck à Berlin; ce dernier avec l'équatorial de m. 0.65 de l'Observatoire de Neubabelsberg (Die Sterne, I937).

A l'Observatoire de Starya Doubossary, en Bessarabie, M. Donitch a constaté que la ligne sombre traversant le disque de la planète n'a pas disparu au moment du passage du plan de celui-ci par le Soleil (L'Astronomie, I937).

M. Quénisset à Juvisy a photographié les inégalités de lumière et d'épaisseur des anneaux. Le 2r juin, c'est à dire une huitaine de jours avant le passage de la Terre par le plan des anneaux, ceux-ci étaient surtout visibles à l'ouest de la planète; à l'est ils étaient très faibles. Le I7 juillet c'est le contraire qui est arrivé. Sur le disque la bande équatoriale de Wood a été très foncée.

Le même observateur a remarqué, les I5 et 23 décembre, des "grains de lumière" sur la ligne de l'anneau (L'Astronomie, 50).

Une communication de M. E. C. Slipher à l'American Astronomical Society se rapporte aux aspects présentés par les anneaux vus par la tranche. Sur ce même sujet $M$. Plakidis nous a envoyé les observations de $M$. Focas à Athènes, d'après lesquelles on déduit que lors de la disparition en juillet 1936 on a vu des condensations près de la division de Cassini. En janvier I937 et jusqu'en février on a pu voir l'anneau et son ombre sur le globe (A.N. 263).

A Collurania nous avons effectué des observations photométriques et visuelles, ces dernières se rapportant surtout à la deuxième disparition de l'anneau en déc. I936-janv. I937. A partir du 27 déc. I936 l'anneau, au lieu de devenir de plus en plus mince, s'élargit aux anses et prit l'aspect de deux points d'exclamation enfoncés dans le globe. La moitié précédente a été toujours bien vue, tandis que la suivante cessa d'être visible environ 4" avant d'atteindre le bord du disque. Lorsque le soleil passa par le plan des anneaux on a vu une sorte de nébulosité envelopper la ligne des anses; cette nébulosité montait des anses jusqu'au bord du disque. L'ombre de l'anneau sur le globe a été vue très souvent double. Les conclusions des observations de Collurania sont:

(a) La présence de matière nébulaire ou corpusculaire en dehors du plan de l'anneau.

(b) La visibilité de la face boréale de l'anneau même dans les époques pendant lesquelles le soleil illuminait la face australe.

Uranus. E. M. Antoniadi a publié une étude sur Uranus dans $L^{\prime}$ Astronomie, 50, étude où il expose l'histoire de la découverte de cette planète, ainsi que les résultats des observations physiques de Laxell, Young, Schiaparelli, Barnard, etc. et enfin par lui-même à Meudon. La grande lunette de Meudon a montré à Antoniadi des 
calottes polaires grisâtres et deux bandes ombrées faibles et un peu ondulées, situées de part et d'autre d'une zone équatoriale claire. Ces bandes étaient à peu près parallèles au plan général des orbites des satellites, et étaient variables comme celles de Jupiter et de Saturne. Cette étude contient aussi un exposé des résultats les plus importants obtenus sur la durée et le sens de la rotation, sur le spectre et sur l'état physique probable de la planète.

\section{RECHERCHES PHYSIQUES}

MM. Pettit et Nicholson ont publié une étude sur la radiation envoyée par la planète Mercure d'après leurs mesures effectuées en I923-25 avec la "vacuum thermocouple" appliquée au réflecteur de Ioo pouces. Les courbes de variation de la radiation calorifique et de la lumière réfléchie avec l'angle de phase ressemblent à celles qu'on obtient pour la Lune. La température au point sub-solaire à la distance moyenne de la planète résulte de $685^{\circ}$ au périhélie et $55^{\circ}$ à l'aphélie. L'albedo radiométrique de Mercure est 0.067 .

N. P. Barabashev et B. E. Semeykine ont continué les études sur l'atmosphère de Venus; ils ont effectué une étude photométrique de Vénus, se basant sur les photographies prises avec trois filtres, rouge, jaune et bleu. La distribution de l'intensité sur le disque de la planète ne s'accorde pas avec la distribution théorique déduite d'après les recherches de Schoenberg. Une particularité intéressante gît dans le fait que, dans les rayons rouges l'intensité maximum pour chaque phase correspond au point où l'angle d'incidence est égal à l'angle de réflexion sur la surface de la planète (Charkow Publ. 5).

M. A. Adel a étudié au point de vue théorique les bandes du spectre infra-rouge attribuées au $\mathrm{CO}_{2}$; il trouve que pour produire les bandes d'absorption de Vénus il faut un parcours de $400 \mathrm{~m}$./atm. Comme il s'agit du parcours aller et retour il faut le réduire à 200 mètres, qui reviennent presque à $100 \mathrm{~m}$./atm. si on considère l'absorption (Astroph. Journ. 85).

Le même auteur est revenu sur le problème de la température de Vénus déduite de la distribution de l'intensité d'absorption dans les bandes du $\mathrm{CO}_{2}$ d'après le spectre de la planète publié par Adams et Dunham Jr. Il trouve que la température de $50^{\circ} \mathrm{C}$., calculée par Menzel d'après les recherches de Coblentz et Lampland en 1922, est très probablement trop petite (Astroph. Journ. 86).

Sur la planète Mars le faible diamètre apparent n'a pas permis de recherches physiques. D'après les informations reçues de M. Dunham Jr. les recherches qu'on a effectuées à ce propos à Mount Wilson pendant ces dernières années ont été celles conduites par lui et Adams sur le spectre de Mars dans l'infra-rouge: on a soupçonné la présence sur Mars de vapeur d'eau, mais on n'a pu en avoir l'évidence. M. Dunham Jr. nous a écrit aussi qu'on a fait à Mount Wilson des essais de photographie des couleurs sur les planètes au foyer de 75 mètres du télescope de roo pouces, sans aucun système d'agrandissement. On a photographié Mars, Jupiter et Saturne sur film Kodachrome de la Eastman Co.

Un essai de mesures photoélectriques des surfaces planétaires a été fait par nous à Collurania pendant l'année I936 avec le photomètre à deux cellules. Sur Jupiter on a mesuré l'intensité de plusieurs taches sombres des bandes tropicales et de la zone équatoriale; sur Saturne on a effectué des mesures le long du diamètre polaire et on a déduit l'intensité des zones et des bandes. Comme les mesures ont été exécutées dans deux radiations différentes, on a déduit l'indice de couleur des différentes régions: pour Jupiter on a $\mathrm{GI}$, pour Saturne $\mathrm{G}_{2}$ pour l'anneau et $\mathrm{G}_{3}$ pour le globe (R. Acc. Lincei, 29). 
Une étude plus détaillée des changements d'intensité de la face de l'anneau tournée vers le Soleil a été effectuée par nous (La Ricerca Scientifica, Anno viII) pendant la période de disparition en 1937. On a déduit: $(a) \mathrm{La}$ brillance de la face boréale de l'anneau a diminué dans les deux groupes de radiations qui correspondent au maximum de sensibilité de deux cellules $\mathrm{Na}$ et $\mathrm{Rb}$. (b) La couleur de l'anneau est demeurée plus blanche que celle des régions équatoriales du globe jusqu'à ce que l'angle d'incidence des rayons solaires a été au-dessous des $88^{\circ} \cdot 5$, après quoi la couleur a tourné vers l'orangé. Le type spectral de l'anneau a tourné du GI à K2.

A propos des mesures photométriques, il faut rappeler les recherches sur l'atmosphère des planètes d'après leurs ombres portées sur les satellites, recherches effectuées par M. Link, qui a publié une note dans les Comptes Rendus, 200, et un mémoire dans le Bulletin Astronomique, 9: d'après ces recherches, tout se passe comme si Jupiter était entouré d'une couche absorbante élévée dont le niveau supérieur est à 20 per cent du rayon. La couche ne serait pas homogène, le maximum de densité serait à une altitude égale au dixième du rayon environ.

Ces études sur les atmosphères planétaires nous montrent tout l'intérêt qu'il y a d̀ appliquer aux corps du système solaire les méthodes de l'astrophysique moderne. Il faut vaincre l'indifférence générale dont la physique planétaire est l'objet. Dans ce même ordre d'idées B. P. Gerasimovich a publié un travail (Bulletin de l'Obs. Centr. Poulkovo, 15) dans lequel il a envisagé le problème de l'illumination d'une planète couverte par une atmosphère épaisse en partant des équations du transport de radiation établies par Eddington pour les étoiles. Dans ce travail Gerasimovich donne une théorie de l'atmosphère de Vénus suivant les variations observées à sa phase, il cherche ensuite dans quel cas-dispersion, absorption ou les deux ensemble--la distribution de l'intensité observée sur les disques de Jupiter et Saturne s'accorde avec la théorie; il cherche aussi une explication aux excès de couleur constatés pour Jupiter et Saturne.

En vue de nous procurer des données sur la distribution de l'intensité sur les disques planétaires, nous avons repris à Collurania les mesures sur les photos de Vénus, Mars et Jupiter effectuées en I929 dans le jaune et dans le bleu; ces mesures, exécutées à l'aide d'un microphotomètre photoélectrique, seront bientôt publiées.

L'état physique de l'atmosphère de Jupiter a été étudié par M. Peek au point de vue des relations entre pression, température et densité; il a considéré trois modèles: adiabatique, isothermique, et un troisième dans lequel est adopté une relation empirique entre hauteur de l'atmosphère et variation de température, dans le cas d'une atmosphère composée de méthane et de méthane et hydrogène (M.N. 97).

M. R. Wildt a publié une note sur la photochimie des atmosphères planétaires. Toutes les molécules polyatomiques constatées dans les atmosphères planétaires sont sensibles à la radiation ultra-violette et peuvent donner lieu à des réactions secondaires. L'existence du méthane et l'impossibilité de déceler les autres hydrocarbures est un signe de l'abondance d'hydrogène dans les grandes planètes et, avec beaucoup de probabilité, il n'y a pas de monoxide ni bioxide de carbone ni $\mathrm{H}_{2} \mathrm{~S}$. M. Wildt remarque que la formaldehyde, trouvée par des moyens chimiques dans l'atmosphère terrestre, possède un spectre d'absorption électronique dans l'ultra-violet accessible aux spectrographes astronomiques; il serait par conséquent intéressant de la rechercher dans le spectre de la planète Vénus (Astroph. Journ. 86).

La chimie des astres a été le sujet d'un compte rendu à l'Académie Royale des 
Lincei par MM. Abetti et Rolla. La composition des atmosphères planétaires y a été traitée, au point de vue des découvertes de Wildt, Dunham, Adel et Slipher (R. Acc. dei Lincei, Problemi e Discussioni, r, I937).

Petites planètes. Les recherches physiques sur les petites planètes par les membres de notre commission ont été peu nombreuses. M. L. Campbell nous a écrit que l'Association Américaine des Observateurs d'étoiles variables a été sollicitée par lui pour la détermination de la magnitude des petites planètes lors de l'opposition, afin d'avoir un contrôle des magnitudes calculées. M. L. Campbell rappelle que l'opposition d'Eros en 1938 pourra fournir des données sur la rotation axiale et sur la duplicité de la planète.

Mlle Harwood a effectué une série d'observations photographiques d'Eros, du I3 octobre I937 au 5 avril 1938 . Les clichés pris pendant 20 nuits, à de courts intervalles, fournissent une courbe de lumière complète, en désaccord avec les prédictions de Watson. Ces observations, insuffisantes pour résoudre le problème, montrent sa complexité et la nécessité de multiplier les observations photographiques et photoélectriques aux oppositions futures.

M. Fletcher Watson Jr. a publié une étude sur la nature physique de Eros (Harvard Cir. No. 4I9); les conditions pour le maximum d'amplitude dans le changement d'éclat y sont discutées, ce qui permet de prévoir l'amplitude pour l'opposition 1938. La forme de la planète est, avec probabilité, un solide allongé et irrégulier et pas un "astre double".

Nous donnons ci-dessous les titres de deux travaux se rapportant à Eros:

"Untersuchungen über Gestalt und Grösse des Planetoiden Eros" von W. Krug und G. Schrutka-Rechtenstamm (Zeitschrift für Astrophysik, 13, I, I936).

"On the Rotation of Eros" by W. Tsesevich (Observatory, 60, 289, 1937).

Satellites. La déclinaison fort australe de Jupiter a empêché toute observation physique sur ses satellites.

Un travail très important a été publié par $\mathrm{E}$. M. Antoniadi sur le troisième satellite de Jupiter (B.A.A.J. 48). Cet observateur analyse les anciens dessins de Dawes, de Lassell, de Barnard etc., de même que ses croquis pris à la grande lunette de Meudon en 1927; il en conclut que la période de rotation de ce satellite est égale à celle de révolution autour de la planète. Le changement dans le profil du disque, tantôt rond et tantôt allongé, serait dû aux taches.

Sur Saturne on a observé le passage de Titan devant le disque lors de la disparition des anneaux.

Mentore Maggini

Président de la Commission 